MUTGEN 01911

\title{
ortho-Substituent effects on the in vitro and in vivo genotoxicity of benzidine derivatives
}

\author{
Z. You, M.D. Brezzell, S.K. Das, M.C. Espadas-Torre, \\ B.H. Hooberman and J.E. Sinsheimer \\ College of Pharmacy, University of Michigan, Ann Arbor, MI 48109-1065, USA
}

(Received 11 December 1992)

(Revision received 26 March 1993)

(Accepted 31 March 1993)

Keywords: 3,3'-Disubstituted benzidine derivatives; Salmonella mutagenicity; Chromosomal aberrations, bone-marrow, in vivo; $\mathrm{pKa}$; LUMO energy; Partition coefficients; Structure-activity relationships, quantitative

\section{Summary}

Benzidine and its 3,3'-diamino, 3,3'-dimethyl, 3,3'-dimethoxy, 3,3'-difluoro, 3,3'-dichloro, 3,3'-dibromo, 3,3'-dicarbomethoxy and 3,3'-dinitro derivatives together with 2-nitrobenzidine and 3-nitrobenzidine were compared for their in vitro and in vivo genotoxicity. Relative mutagenicity was established with Salmonella strains TA98, TA98/1,8-DNP 6 and TA100 with and without 59 activation. All the derivatives in the presence of $\mathbf{S 9}$ were more mutagenic than benzidine with 3,3'-dinitro- and 3-nitro-benzidine having the greatest mutagenicity. Mutagenicity in all 3 strains with $S 9$ activation could be correlated to electron-withdrawing ability of substituent groups, as measured by the basicity of the amines. This correlation was explained on the basis that electron-withdrawing groups could favor the stability of the mutagenic intermediate $N$-hydroxylamine and also enhance the reactivity of the ultimate mutagenic species, the nitrenium ion. Mutagenicity was also correlated to the energy of the lowest unoccupied molecular orbitals $\left(E_{\mathrm{LUMO}}\right)$. Hydrophobicity was found to have very limited effect on the relative mutagenicity of our benzidine derivatives. The in vivo endpoint was chromosomal aberrations in the bone-marrow cells of mice following intraperitoneal administration of benzidine and its derivatives. In contrast to the in vitro results, while all the amines were genotoxic in vivo, only the 3-nitro derivative had a significant increase in toxicity over benzidine.

Aromatic amines are widely used industrial and laboratory reagents and since it is well established that such compounds can be activated in vivo to form DNA adducts, the genotoxicity of these amines is of concern (Weisburger and

Correspondence: Dr. J.E. Sinsheimer, College of Pharmacy, University of Michigan, Ann Arbor, MI 48109-1065, USA.
Thorgeirsson, 1981). Thus, there is a growing interest in examining quantitative structure-activity relationships (QSAR) for the genotoxicity of aromatic amines. Most of these QSAR studies use Salmonella mutagenicity data as their measure of genotoxicity with the objective of developing predictive models for in vivo toxicity (Kalopissis, 1991; Debnath et al., 1992; Ford and Herman, 1992; Ford and Griffin, 1992). The work of Sabbioni (1992) using hemoglobin binding of mono- 
cyclic aromatic amines is an extension of in vitro QSAR studies to an in vivo model.

Our interest in structure-activity studies of aromatic amines is focused on compounds related to the known human carcinogen, benzidine (Messerly et al., 1987; Sinsheimer et al., 1992). The first of these studies indicated a possible correlation between the mutagenicity of benzidine derivatives and their basicity as affected by ortho substitution (Fujita and Nishioka, 1976; Segura, 1985). The second study showed for benzidine and 12 related aromatic amines that both the electronic effects of substituents and the degree of conjugation between phenyl rings in a compound were major determinants of the in vitro Salmonella mutagenicity of these amines, but that mutagenicity was not predictive of relative in vivo genotoxicity.

It is the purpose of the present investigation to determine quantitatively substituent electronic (donating/withdrawing) effects on the mutagenicity of benzidine derivatives and their related compounds in Salmonella strains TA98, TA98/1,8-- - NP $_{6}$ and TA100. In addition, it is also of interest to examine the correlation of mutagenicity with frontier orbital energy (Debnath et al., 1992; Sabbioni, 1992) as well as hydrophobicity (Debnath et al., 1992) of these compounds. For the objective of comparing in vitro and in vivo genotoxicities, chromosomal aberrations (CA) in the bone-marrow cells of mice were determined following intraperitoneal (i.p.) administration of the amines. Test compounds in this paper include benzidine and 8 of its $3,3^{\prime}$-disubstituted derivatives which were selected to examine ortho effects on mutagenicity. 2-Nitro- and 3-nitro-benzidine were also included in the studies for comparison to the genotoxicity of $3,3^{\prime}$-dinitrobenzidine. In a following paper, correlation studies of substituent effects on the genotoxicity of 4-aminobiphenyl and 4-aminostilbene derivatives are presented (You et al., 1993).

\section{Materials and methods}

\section{Test compounds}

Benzidine (92-87-5) was purchased from Harleco, Philadelphia, PA, while 3,3'-dimethoxybenzidine (119-90-4), 3,3'-diaminobenzidine (91-
95-2) and the positive control, 7,12-dimethylbenz $[a]$ anthracene (DMBA) (57-97-6), were obtained from Aldrich Chemical Co., Milwaukee, WI. 3,3'-Dichlorobenzidine dihydrochloride was purchased from Sigma, St. Louis, MO. This salt was treated with aqueous sodium hydroxide to yield 3,3'-dichlorobenzidine (91-94-1): m.p. 132$133^{\circ} \mathrm{C}$ (Buckingham, 1982: $133^{\circ} \mathrm{C}$ ).

3,3-Dicarbomethoxybenzidine (15403-45-9) was obtained by treatment of benzidine-3,3'-dicarboxylic acid (Pfaltz and Bauer, Waterbury, CT) in refluxing methanol containing concentrated sulfuric acid for 2 days. A purified sample (22\%, green-grey powder) was obtained through crystallization from ethyl acetate/methanol: m.p. 211-212 $2^{\circ} \mathrm{C}$ (Iwakura et al., 1973: $212^{\circ} \mathrm{C}$ ). Proton NMR $\left(270 \mathrm{MHz}, \mathrm{CDCl}_{3}\right): 8.032(2 \mathrm{H}, \mathrm{d}, J=2.0$ $\mathrm{Hz}), 7.492(2 \mathrm{H}, \mathrm{dd}, J=8.6,2.0 \mathrm{~Hz}), 6.731(2 \mathrm{H}, \mathrm{br}$ s), $3.908(6 \mathrm{H}, \mathrm{s})$.

3,3'-Dimethylbenzidine (119-93-7) was prepared by reduction of 3,3'-dicarbomethoxybenzidine with lithium aluminum hydride ( $>10$ molar equiv.) in refluxing diethyl ether for 1 day. Purification was performed through silica gel column chromatography with hexanes/ethyl acetate (2:1) as solvent to yield a beige-grey powder (24\%): m.p. $123.5-125.5^{\circ} \mathrm{C}$ (Weast and Grasselli, 1989: $\left.131-132^{\circ} \mathrm{C}\right)$. Proton NMR (270 $\mathrm{MHz}, \mathrm{CDCl}_{3}$ ): $7.242(2 \mathrm{H}, \mathrm{s}), 7.227(2 \mathrm{H}, \mathrm{d}, J=8.0 \mathrm{~Hz}), 6.713$ $(2 \mathrm{H}, \mathrm{d}, J=8.0 \mathrm{~Hz}), 3.606(4 \mathrm{H}, \mathrm{br} \mathrm{s}), 2.220(6 \mathrm{H}, \mathrm{s})$.

3,3'-Difluorobenzidine (448-97-5) was synthesized through benzidine rearrangement of 2,2'hydrazine difluorobenzene which in turn was derived from 2-fluorobenzene (Savard and Josephy, 1986). It was purified by silica gel column chromatography with hexanes/ethyl acetate $(2: 1)$ as solvent to give a brownish powder: m.p. 116$118^{\circ} \mathrm{C}$ (Savard and Josephy, 1986: $119-120^{\circ} \mathrm{C}$ ). Proton NMR ( $\left.300 \mathrm{MHz}, \mathrm{CDCl}_{3}\right): 7.156(2 \mathrm{H}$, dd, $J=12.4,2.0 \mathrm{~Hz}), 7.100(2 \mathrm{H}, \mathrm{dd}, J=8.0,2.0 \mathrm{~Hz})$, $6.797(2 \mathrm{H}, \mathrm{dd}, J=9.2,8.0 \mathrm{~Hz}), 3.731(4 \mathrm{H}$, br s).

2-Nitrobenzidine (2243-78-9) was obtained by treatment of benzidine with nitric acid/sulfuric acid (Leslie and Turner, 1933): m.p. $142-143^{\circ} \mathrm{C}$ (Buckingham, 1982: $143^{\circ} \mathrm{C}$ ). Proton NMR (270 $\left.\mathrm{MHz}, \mathrm{CDCl}_{3}\right): 7.177(1 \mathrm{H}, \mathrm{d}, J=8.3 \mathrm{~Hz}), 7.061$ $(2 \mathrm{H}, \mathrm{d}, J=8.6 \mathrm{~Hz}), 7.045(1 \mathrm{H}, \mathrm{d}, J=2.5 \mathrm{~Hz})$, $6.839(1 \mathrm{H}, \mathrm{dd}, J=8.3,2.5 \mathrm{~Hz}), 6.690(2 \mathrm{H}, \mathrm{d}$, $J=8.6 \mathrm{~Hz}), 3.916(2 \mathrm{H}$, br s$), 3.728(2 \mathrm{H}$, br s$)$. 
Benzidine was treated with an excess of acetic anhydride (15 equiv) and $N, N^{\prime}$-diacetylbenzidine (white powder) was obtained quantitatively by filtration, washing with water and drying under vacuum: m.p. $329-332^{\circ} \mathrm{C}$ (Weast and Grasselli, 1989: $330-331^{\circ} \mathrm{C}$ ). This product was used to synthesize the following benzidines.

3,3'-Dibromobenzidine (34237-98-4) was prepared from bromination of $N, N^{\prime}$-diacetylbenzidine followed by hydrolysis (Snyder et al., 1949), and purified through silica gel chromatography with hexanes/ethyl acetate $(3: 2)$ to yield a beige solid: m.p. $128.5-130.5^{\circ} \mathrm{C}$ (Savard and Josephy, 1986: $\left.129-131^{\circ} \mathrm{C}\right)$. NMR ( $\left.300 \mathrm{MHz}, \mathrm{CDCl}_{3}\right): 7.565$ $(2 \mathrm{H}, \mathrm{d}, J=2.1 \mathrm{~Hz}), 7.250(2 \mathrm{H}, \mathrm{dd}, J=8.3,2.1$ $\mathrm{Hz}), 6.792(2 \mathrm{H}, \mathrm{d}, J=8.3 \mathrm{~Hz}), 4.097(4 \mathrm{H}, \mathrm{br} \mathrm{s})$.

3-Nitrobenzidine (61841-39-2) was prepared by treatment of $N, N^{\prime}$-diacetylbenzidine with nitric acid/acetic acid (Mysyk, 1976) followed by hydrolysis in refluxing aqueous sodium hydroxide. The product was purified by silica gel chromatography with dichloromethane to yield a red solid: m.p. 213 $-214^{\circ} \mathrm{C}$ (Buckingham, 1982: $215^{\circ} \mathrm{C}$ ). NMR (270 $\left.\mathrm{MHz}, \mathrm{CDCl}_{3}\right): 8.292(1 \mathrm{H}, \mathrm{d}, J=2.2 \mathrm{~Hz}), 7.593$ $(1 \mathrm{H}, \mathrm{dd}, J=8.6,2.2 \mathrm{~Hz}), 7.372(2 \mathrm{H}, \mathrm{d}, J=8.5$ $\mathrm{Hz}), 6.856(1 \mathrm{H}, \mathrm{d}, J=8.6 \mathrm{~Hz}), 6.755(2 \mathrm{H}, \mathrm{d}$, $J=8.5 \mathrm{~Hz}), 6.053(2 \mathrm{H}$, br s), $3.753(2 \mathrm{H}$, br s$)$.

3,3'-Dinitrobenzidine (6271-79-0) was synthesized by nitration of $N, N^{\prime}$-diacetylbenzidine according to the procedure of Barker and Casson (1953). The product was recrystallized from pyridine/ethyl acetate to give red crystals: m.p. 275$276^{\circ} \mathrm{C}$ (Buckingham, 1982: $276^{\circ} \mathrm{C}$ ). NMR (270 $\left.\mathrm{MHz}, \mathrm{CDCl}_{3}\right): 8.322(2 \mathrm{H}, \mathrm{d}, J=2.1 \mathrm{~Hz}), 7.624$ $(2 \mathrm{H}, \mathrm{dd}, J=8.6,2.1 \mathrm{~Hz}), 6.908(2 \mathrm{H}, \mathrm{d}, J=8.6$ $\mathrm{Hz}), 6.146(4 \mathrm{H}, \mathrm{br} \mathrm{s})$.

All test compounds at $0.01 \mu$ mole showed a single spot under UV light on Silica-GF Uniplates (Analtech Inc., Newark, DE) after development with hexanes/ethyl acetate solvent systems. This together with the melting point and NMR data above as well as the chromatograms developed for the determination of partition coefficients indicated that the test compounds were of $>98 \%$ purity.

\section{Mutagenicity assays}

Compounds dissolved in DMSO were tested for concentration-mutagenicity relationships us- ing Salmonella typhimurium strains TA100 and TA98 kindly provided by Dr. B.N. Ames, University of California, Berkeley as well as strain TA98/1,8-DNP 6 kindly provided by Dr. H.S. Rosenkranz, University of Pittsburgh. The standard plate-incorporation assay with and without metabolic activation as described by Maron and Ames (1983) was used. Plates were scored with an automatic counter (Autocount, Dynatech Labs, Chantilly, VA) which had been calibrated against manually scored plates. Each dose was run in triplicate and the dose-response relationship confirmed in a second set of tests. The linear range of the dose-response curve for each amine was determined using the statistical procedures of Bernstein et al. (1982).

\section{Chromosomal aberrations}

Test chemicals were administered i.p. in DMSO $(2 \mathrm{ml} / \mathrm{kg})$ as single $100 \mathrm{mg} / \mathrm{kg}$ doses to each of 4 mice. Negative control mice were injected with $2 \mathrm{ml} / \mathrm{kg}$ DMSO while DMBA was used as a positive control at a dose of $100 \mathrm{mg} / \mathrm{kg}$ in DMSO. The dose for the test compounds is based upon our concentration-response data reported for benzidine (Sinsheimer et al., 1992). CA assays were conducted as previously described (Giri et al., 1989) with a scoring time of $24 \mathrm{~h}$ after injection which is consistent with the protocol of Preston et al. (1987). This scoring time was found to be an optimal time for benzidine after testing at 6,12 and $24 \mathrm{~h}$. All the slides were coded and observed by a single observer where 100 well-spread metaphase cells were scored per animal from each of 4 animals at each concentration tested. Mitotic indices were calculated from 1000 cells/animal and expressed as percentages. The aberration frequencies per cell for chromatid and chromosome types were calculated. Gaps were recorded but not included in the frequency of aberrations per cell.

\section{Relative partition coefficients}

High-performance liquid chromatography (HPLC) was carried out with a C-18 reverse-phase column (Partisil PXS 10/25 ODS-3, Whatman, Clifton, $\mathrm{NJ}$ ) and solvent mixture of water and methanol (45:55 with $0.1 \%$ ammonium formate) at a flow rate of $1 \mathrm{ml} / \mathrm{min}$. Samples were dis- 
solved in methanol for injection and all retention times were confirmed by a second experiment. The partition coefficients were calculated by the method of Carlson et al. (1975) using the relationship $\pi_{\text {HPLC }}=\log k_{\text {derivative }}^{\prime}-\log k_{\text {benzidine }}^{\prime}$ where $k^{\prime}=($ retention time - void volume)/void volume.

\section{Frontier orbital energies}

All calculations were carried out on a Silicon Graphics computer with IRIS 4D/360GTX central processor. The compounds were geometryoptimized by QUANTA programs with CHARMm force field (QUANTA release 3.2, Polygen Corp.). These energy-minimized structures were then used to calculate the frontier orbital energies ( $E_{\text {Hомо }}$ and $E_{\text {LUMO }}$ ) through PM3 approximation (Steward, 1989a,b) of the MOPAC 6.0 package (Quantum Chemistry Program Exchange, Indiana University, Indiana) without further geometry-optimization. Each compound had at least two conformations with energy near the global minimum. Of these conformations, only the frontier orbital energies for the structure with the lowest heat of formation were taken for the correlations.

\section{Results and discussion}

\section{In vitro mutagenicity}

The Salmonella mutagenicity results with and without S9 activation in strains TA98, TA98/1,8$\mathrm{DNP}_{6}$ and TA100 for benzidine and its derivatives are summarized in Table 1. Each of the corresponding slopes (revertants $/ \mu$ mole) in Table 2 were obtained from the linear portion of the dose-response curve derived from the data in Table 1. As was to be expected, based upon the well established metabolic activation of aromatic amines to produce DNA alkylating agents (Beland and Kadlubar, 1985, 1990), mutagenicity was greater for all compounds in the presence of rat-liver S9 fractions than in its absence. However, most of the compounds had measurable activity without S9 activation in strain TA98 and with some of the compounds this was also true in strains TA98/1,8-DNP 6 and TA100. The halogenated benzidines had the most pronounced activity without $S 9$ activation in relationship to the response following activation. Savard and Josephy (1986) cited this activity as evidence for multiple mechanisms for the genotoxicity of these compounds. Even with the halogenated benzidines, the majority of the mutagenicity resulted from amino group activation.

The results with strain TA98/1,8-DNP 6 , with its absence of $O$-acetyltransferase (Orr et al., 1985; Saito et al., 1985), showed reduced mutagenicity compared to strain TA98. This decrease was not consistent among compounds, with the 3,3'-dimethoxy derivative and benzidine having the greatest percentage loss and 3,3'-dinitrobenzidine retaining most of its activity. However, the correlation coefficient $\left(r^{2}\right)$ between the log of mutagenicity of TA98 and TA98/1,8-DNP ${ }_{6}$ for the 11 compounds in Table 1 is high (0.92). This is also true for the comparison of TA98 to TA100 (0.83), although in the latter series there were lower levels of activity.

In Table 3 are listed the $\mathrm{p} K$ a values of $o$-substituted anilines corresponding to our $3,3^{\prime}$-disubstituted benzidines, experimentally derived $\mathrm{p} K \mathrm{a}$ values for some of the test compounds, relative partition coefficient $\left(\pi_{\text {HPLC }}\right)$ values and frontier orbital energies $\left(E_{\text {HOMO }}\right.$ and $E_{\text {LUMO }}$ ) of the benzidine derivatives. The aniline values are included as an indication of relative basicity as there is only limited basicity data for the benzidines (Zheltov et al., 1970). We were able to measure the $\mathrm{pKa}$ values of benzidine and 4 of its derivatives by a spectrophotometric method (Albert and Serjeant, 1984), but the limited solubility of 3,3'-diamino-, 3,3'-dicarbomethoxy-, and 3,3'dinitro-benzidine prevented their determination. Although the values found for these 5 compounds are larger than those of their structurally similar anilines, these two measures of basicity data correlate well $\left(r^{2}=0.94\right)$.

3-Nitrobenzidine was included in all our mutagenicity correlations but 2-nitrobenzidine was not. The 2-nitro derivative was excluded since it has a different geometry and its conjugation between the two phenyl rings is reduced by steric interference of the nitro group. This compound is more mutagenic than benzidine which could be explained by the electronic effect of the nitro substituent counteracting the mutagenicity-reducing effect of the loss of conjugation (Messerly et al., 
TABLE 1

MUTAGENICITY OF BENZIDINES IN Salmonella typhimurium a

\begin{tabular}{|c|c|c|c|c|c|c|}
\hline \multirow{2}{*}{$\begin{array}{l}\text { Compounds } \\
\text { ( } \mu \text { moles) }\end{array}$} & \multicolumn{2}{|l|}{ TA98 } & \multicolumn{2}{|c|}{ TA98/1,8-DNP 6} & \multicolumn{2}{|l|}{ TA100 } \\
\hline & $-\mathbf{S} 9$ & $+\mathbf{S} 9$ & $-\mathrm{S9}$ & $+\$ 9$ & -59 & $+\mathbf{S 9}$ \\
\hline \multicolumn{7}{|l|}{ Benzidine } \\
\hline 0 & $29 \pm 6$ & $40 \pm$ & $41 \pm$ & $60 \pm 12$ & $121 \pm 22$ & $151 \pm 15$ \\
\hline 0.1 & $32 \pm 12$ & $56 \pm 9$ & $36 \pm$ & $48 \pm 11$ & $132 \pm 21$ & $146 \pm 23$ \\
\hline 0.25 & $24 \pm 2$ & $87 \pm 11$ & & & $140 \pm 11$ & $183 \pm 11$ \\
\hline 0.5 & $29 \pm 10$ & $118 \pm 10$ & $37 \pm 5$ & $63 \pm 5$ & $137 \pm 20$ & $181 \pm 30$ \\
\hline 1 & $24 \pm 6$ & $149 \pm 32$ & $39 \pm$ & $61 \pm 17$ & $128 \pm 15$ & $217 \pm 31$ \\
\hline 2.5 & $29 \pm 8$ & $229 \pm 19$ & & & $153 \pm 23$ & $258 \pm 10$ \\
\hline 5 & $27 \pm 4$ & $357 \pm 35$ & $38 \pm 12$ & $69 \pm 11$ & $126 \pm 11$ & $242 \pm 22$ \\
\hline 10 & $27 \pm 5$ & $472 \pm 14$ & $41 \pm 8$ & $94 \pm 10$ & $116 \pm 15$ & $239 \pm 25$ \\
\hline \multicolumn{7}{|c|}{ 3,3'-Diaminobenzidine } \\
\hline 0 & $36 \pm 2$ & $57 \pm 14$ & $30 \pm$ & $36 \pm 7$ & $106 \pm 8$ & $129 \pm 10$ \\
\hline 0.01 & $42 \pm 3$ & $80 \pm 31$ & $28 \pm$ & $46 \pm 20$ & $91 \pm 11$ & $143 \pm 19$ \\
\hline 0.05 & $46 \pm 8$ & $223 \pm 31$ & $30 \pm$ & $66 \pm 18$ & $99 \pm 12$ & $168 \pm 14$ \\
\hline 0.1 & $40 \pm 9$ & $318 \pm 19$ & $35 \pm 8$ & $124 \pm 16$ & $106 \pm 15$ & $209 \pm 15$ \\
\hline 0.5 & $50 \pm 9$ & $752 \pm 61$ & $33 \pm 13$ & $192 \pm 8$ & $145 \pm 21$ & $293 \pm 15$ \\
\hline 1 & $55 \pm 19$ & $948 \pm 100$ & $33 \pm 10$ & $285 \pm 10$ & $180 \pm 12$ & $346 \pm 29$ \\
\hline \multicolumn{7}{|c|}{ 3,3'-Dimethoxybenzidine } \\
\hline 0 & $29 \pm 8$ & $40 \pm 12$ & $28 \pm 3$ & $40 \pm 8$ & $104 \pm 16$ & $135 \pm 21$ \\
\hline 0.01 & $28 \pm 11$ & $98 \pm 40$ & & & $122 \pm 9$ & $131 \pm 6$ \\
\hline 0.05 & $31 \pm 9$ & $195 \pm 25$ & & & $111 \pm 16$ & $146 \pm 19$ \\
\hline 0.1 & $31 \pm 12$ & $277 \pm 51$ & $29 \pm 6$ & $54 \pm 9$ & $113 \pm 15$ & $150 \pm 20$ \\
\hline 0.2 & $27 \pm 12$ & $428 \pm 73$ & & & $119 \pm 17$ & $156 \pm 12$ \\
\hline 0.33 & & & $36 \pm 4$ & $70 \pm 10$ & & \\
\hline 0.4 & $25 \pm 7$ & $541 \pm 76$ & & & $104 \pm 13$ & $182 \pm 21$ \\
\hline 0.5 & $55 \pm 10$ & $614 \pm 29$ & & & $114 \pm 15$ & $195 \pm 11$ \\
\hline 0.66 & & & $39 \pm 6$ & $82 \pm 15$ & & \\
\hline 0.8 & & & & & $123 \pm 29$ & $192 \pm 29$ \\
\hline 1 & $30 \pm 1$ & $692 \pm 15$ & $50 \pm 10$ & $99 \pm 31$ & $102 \pm 3$ & $207 \pm 37$ \\
\hline 3 & & & $33 \pm 7$ & $98 \pm 22$ & & \\
\hline \multicolumn{7}{|c|}{ 3,3'-Dimethylbenzidine } \\
\hline 0 & $31 \pm 17$ & $53 \pm 25$ & $28 \pm 3$ & $40 \pm 8$ & $117 \pm 16$ & $126 \pm 17$ \\
\hline 0.05 & & & $27 \pm 6$ & $57 \pm 1$ & & \\
\hline 0.1 & $20 \pm 7$ & $171 \pm 9$ & $28 \pm 7$ & $70 \pm 3$ & $92 \pm 5$ & $138 \pm 4$ \\
\hline 0.33 & $37 \pm 24$ & $344 \pm 44$ & $26 \pm 9$ & $107 \pm 20$ & $120 \pm 18$ & $157 \pm 7$ \\
\hline 0.66 & $40 \pm 10$ & $635 \pm 42$ & $29 \pm 3$ & $160 \pm 1$ & $106 \pm 1$ & $208 \pm 25$ \\
\hline 1 & $45 \pm 16$ & $957 \pm 102$ & $26 \pm 5$ & $272 \pm 75$ & $112 \pm 24$ & $243 \pm 30$ \\
\hline 1.5 & $46 \pm 17$ & $1392 \pm 165$ & & & $103 \pm 10$ & $278 \pm 14$ \\
\hline 2 & $51 \pm 12$ & $1936 \pm 135$ & & & $109 \pm 18$ & $335 \pm 16$ \\
\hline \multicolumn{7}{|c|}{ 3,3'-Difluorobenzidine } \\
\hline 0 & $33 \pm 7$ & $52 \pm 5$ & $47 \pm 13$ & $50 \pm 10$ & $145 \pm 8$ & $150 \pm 24$ \\
\hline 0.01 & $40 \pm 5$ & $124 \pm 23$ & $75 \pm 37$ & $66 \pm 35$ & $135 \pm 1$ & $197 \pm 23$ \\
\hline 0.033 & $47 \pm 8$ & $274 \pm 21$ & $81 \pm 28$ & $91 \pm 24$ & $139 \pm 15$ & $198 \pm 22$ \\
\hline 0.05 & $104 \pm 9$ & & & & $131 \pm 17$ & $203 \pm 11$ \\
\hline 0.066 & $93 \pm 29$ & $437 \pm 39$ & $151 \pm 108$ & $126 \pm 23$ & $153 \pm 16$ & $185 \pm 10$ \\
\hline 0.1 & $95 \pm 33$ & $592 \pm 60$ & $184 \pm 101$ & $163 \pm 22$ & $144 \pm 13$ & $230 \pm 24$ \\
\hline 0.3 & $113 \pm 35$ & $1350 \pm 236$ & $129 \pm 57$ & $353 \pm 41$ & $158 \pm 18$ & $235 \pm 28$ \\
\hline 0.33 & $156 \pm 15$ & & & & & \\
\hline 0.5 & & & & & $125 \pm 7$ & $348 \pm 46$ \\
\hline 0.66 & $201 \pm 13$ & & & & & \\
\hline 1 & $231 \pm 37$ & & & & $136 \pm 16$ & $394 \pm 18$ \\
\hline
\end{tabular}


TABLE 1 (continued)

\begin{tabular}{|c|c|c|c|c|c|c|c|}
\hline \multirow{2}{*}{$\begin{array}{l}\text { Compounds } \\
\text { ( } \mu \text { moles) }\end{array}$} & \multicolumn{2}{|l|}{ TA98 } & \multicolumn{3}{|c|}{$\mathrm{TA}^{2} / 1,8-\mathrm{DNP}_{6}$} & \multicolumn{2}{|l|}{ TA100 } \\
\hline & $-S 9$ & $+\mathrm{S} 9$ & $-\mathrm{S9}$ & $+\$ 9$ & & $-S 9$ & $+\mathrm{S} 9$ \\
\hline \multicolumn{8}{|c|}{ 3,3'-Dichlorobenzidine } \\
\hline 0 & $25 \pm 6$ & $44 \pm 17$ & $20 \pm 4$ & $35 \pm$ & 7 & $107 \pm 15$ & $117 \pm 15$ \\
\hline 0.01 & $92 \pm 29$ & $234 \pm 28$ & $55 \pm 10$ & $114 \pm$ & 41 & & \\
\hline 0.02 & $203 \pm 81$ & $482 \pm 58$ & & & & & \\
\hline 0.03 & $308 \pm 29$ & $743 \pm 102$ & & & & & \\
\hline 0.033 & & & $114 \pm 29$ & $362 \pm$ & 70 & $107 \pm 14$ & $145 \pm 28$ \\
\hline 0.04 & $297 \pm 135$ & $1069 \pm 80$ & & & & & \\
\hline 0.05 & $158 \pm 22$ & $1371 \pm 103$ & & & & & \\
\hline 0.066 & & & $153 \pm 17$ & $676 \pm$ & 106 & $109 \pm 12$ & $186 \pm 21$ \\
\hline 0.1 & $223 \pm 12$ & & $198 \pm 92$ & $1142 \pm$ & 299 & $116 \pm 10$ & $256 \pm 70$ \\
\hline 0.2 & & & & & & $117 \pm 12$ & $388 \pm 48$ \\
\hline 0.3 & & & $325 \pm 55$ & $1626 \pm$ & 175 & $116 \pm 5$ & $509 \pm 47$ \\
\hline 0.33 & $360 \pm 5$ & & & & & & \\
\hline 0.66 & $664 \pm 16$ & & & & & & \\
\hline 1 & $469 \pm 101$ & & & & & & \\
\hline \multicolumn{8}{|c|}{ 3,3'-Dibromobenzidine } \\
\hline 0 & $26 \pm 5$ & $46 \pm 17$ & $25 \pm 8$ & $32 \pm$ & 5 & $136 \pm 24$ & $153 \pm 15$ \\
\hline 0.005 & $57 \pm 5$ & $140 \pm 22$ & & & & & \\
\hline 0.01 & $59 \pm 8$ & $267 \pm 10$ & $38 \pm 3$ & $114 \pm$ & 7 & & \\
\hline 0.02 & $101 \pm 27$ & $529 \pm 20$ & & & & & \\
\hline 0.03 & $122 \pm 29$ & $961 \pm 39$ & & & & & \\
\hline 0.033 & & & $71 \pm 26$ & $384 \pm$ & 28 & $154 \pm 11$ & $206 \pm$ \\
\hline 0.04 & $203 \pm 83$ & $1322 \pm 76$ & & & & & \\
\hline 0.05 & $132 \pm 18$ & $1685 \pm 81$ & & & & & \\
\hline 0.066 & & & $98 \pm 29$ & $904 \pm$ & 163 & $142 \pm 17$ & $202 \pm 41$ \\
\hline 0.1 & $171 \pm 10$ & & $133 \pm 28$ & $1298 \pm$ & 161 & $140 \pm 21$ & $250 \pm 62$ \\
\hline 0.2 & & & & & & $156 \pm 24$ & $371 \pm 89$ \\
\hline 0.3 & & & $214 \pm 49$ & $2113 \pm 1$ & 1006 & $155 \pm 29$ & $500 \pm 119$ \\
\hline 0.33 & $329 \pm 16$ & & & & & & \\
\hline 0.5 & & & & & & $141 \pm 10$ & $683 \pm 24$ \\
\hline 0.66 & $135 \pm 34$ & & & & & & \\
\hline 1 & $73 \pm 27$ & & & & & & \\
\hline \multicolumn{8}{|c|}{ 3,3'-Dicarbomethoxybenzidine } \\
\hline 0 & $31 \pm 9$ & $46 \pm 12$ & $34 \pm 12$ & $51 \pm$ & 17 & $123 \pm 25$ & $142 \pm 26$ \\
\hline 0.005 & $24 \pm 8$ & $175 \pm 41$ & $32 \pm 6$ & $74 \pm$ & 10 & $133 \pm 17$ & $165 \pm 15$ \\
\hline 0.01 & $28 \pm$ & $391 \pm 76$ & $40 \pm 10$ & $117 \pm$ & 21 & $134 \pm 27$ & $166 \pm 22$ \\
\hline 0.02 & $25 \pm$ & $716 \pm 79$ & $37 \pm 7$ & $166 \pm$ & 21 & $117 \pm 13$ & $178 \pm 8$ \\
\hline 0.033 & $43 \pm$ & $1369 \pm 40$ & $29 \pm 9$ & $225 \pm$ & 29 & $148 \pm 21$ & $189 \pm 11$ \\
\hline 0.04 & $30 \pm$ & $1286 \pm 134$ & $43 \pm 10$ & $370 \pm$ & 43 & $125 \pm 10$ & $192 \pm 27$ \\
\hline 0.06 & $33 \pm$ & $1413 \pm 66$ & $41 \pm 9$ & $331 \pm$ & 114 & $103 \pm 10$ & $214 \pm 24$ \\
\hline 0.066 & $39 \pm$ & $1713 \pm 158$ & $30 \pm 6$ & $400 \pm$ & 51 & $135 \pm 17$ & $189 \pm 15$ \\
\hline 0.1 & $51 \pm$ & $1602 \pm 27$ & $22 \pm 9$ & $367 \pm$ & 43 & $138 \pm 19$ & $166 \pm 15$ \\
\hline 0.3 & & & $29 \pm 7$ & $239 \pm$ & 12 & & \\
\hline
\end{tabular}


TABLE 1 (continued)

\begin{tabular}{|c|c|c|c|c|c|c|c|}
\hline \multirow{2}{*}{$\begin{array}{l}\text { Compounds } \\
\text { ( } \mu \text { moles) }\end{array}$} & \multicolumn{3}{|l|}{ TA98 } & \multicolumn{2}{|c|}{$\mathrm{TA} 98 / 1,8-\mathrm{DNP}_{6}$} & \multicolumn{2}{|l|}{ TA100 } \\
\hline & $-\mathrm{S9}$ & & $+\mathrm{S} 9$ & $-\mathrm{S} 9$ & $+\mathbf{S 9}$ & $-S 9$ & $+\mathrm{S} 9$ \\
\hline \multicolumn{8}{|c|}{ 3,3'-Dinitrobenzidine } \\
\hline 0 & $33 \pm$ & 6 & $57 \pm 11$ & $26 \pm 9$ & $25 \pm 7$ & $119 \pm 7$ & $138 \pm 17$ \\
\hline 0.0005 & $37 \pm$ & 6 & $176 \pm 20$ & $30 \pm 8$ & $175 \pm 25$ & $126 \pm 10$ & $171 \pm 19$ \\
\hline 0.001 & $37 \pm$ & 7 & $292 \pm 23$ & $26 \pm$ & $270 \pm 26$ & $125 \pm 18$ & $233 \pm 53$ \\
\hline 0.002 & $39 \pm$ & 2 & $572 \pm 15$ & & & & \\
\hline 0.033 & $30 \pm$ & 4 & $979 \pm 35$ & $32 \pm 11$ & $862 \pm 57$ & $135 \pm 30$ & $443 \pm 100$ \\
\hline 0.004 & $42 \pm$ & 5 & $1279 \pm 117$ & & & & \\
\hline 0.006 & $46 \pm$ & 8 & $2104 \pm 126$ & & & & \\
\hline 0.0066 & $29 \pm$ & 7 & $2379 \pm 88$ & $34 \pm 11$ & $2163 \pm 196$ & $147 \pm 17$ & $916 \pm 192$ \\
\hline 0.01 & $41 \pm$ & 8 & $3585 \pm 28$ & $34 \pm 10$ & $3038 \pm 377$ & $159 \pm 19$ & $1215 \pm 289$ \\
\hline 0.05 & $76 \pm 1$ & 17 & & & & & \\
\hline 0.1 & $88 \pm 1$ & 12 & & & & & \\
\hline 0.5 & $162 \pm 1$ & 17 & & & & & \\
\hline 1 & $237 \pm 3$ & 31 & & & & & \\
\hline \multicolumn{8}{|c|}{ 3-Nitrobenzidine } \\
\hline 0 & $36 \pm$ & 6 & $49 \pm 18$ & $29 \pm 10$ & $36 \pm 7$ & $116 \pm 3$ & $158 \pm 20$ \\
\hline 0.0001 & & & $113 \pm 16$ & & & & \\
\hline 0.00033 & & & $255 \pm 10$ & & & & \\
\hline 0.0005 & $42 \pm$ & 1 & $416 \pm 16$ & $35 \pm 3$ & $71 \pm 30$ & & \\
\hline 0.00066 & & & $543 \pm 15$ & & & & \\
\hline 0.001 & $38 \pm$ & 8 & $820 \pm 73$ & $70 \pm 25$ & $164 \pm 51$ & & $209 \pm 20$ \\
\hline 0.002 & $68 \pm$ & 4 & $1891 \pm 141$ & & & & \\
\hline 0.0025 & & & & $80 \pm 38$ & $287 \pm 16$ & & \\
\hline 0.003 & $79 \pm$ & 9 & $3025 \pm 91$ & & & & \\
\hline 0.0033 & & & & & & & $231 \pm 11$ \\
\hline 0.004 & $94 \pm$ & 6 & $3524 \pm 241$ & & & & \\
\hline 0.005 & $60 \pm 1$ & 10 & & $97 \pm 67$ & $773 \pm 150$ & & \\
\hline 0.0066 & & & & & & & $341 \pm 37$ \\
\hline 0.01 & $80 \pm 2$ & 25 & & $205 \pm 189$ & $1405 \pm 270$ & & $473 \pm 28$ \\
\hline 0.033 & & & & & & & $660 \pm 63$ \\
\hline 0.05 & $244 \pm 1$ & 15 & & $162 \pm 12$ & & $138 \pm 10$ & \\
\hline 0.1 & $351 \pm 5$ & 55 & & $257 \pm 10$ & & $148 \pm 9$ & \\
\hline 0.33 & & & & & & $163 \pm 17$ & \\
\hline 0.5 & $894 \pm 20$ & & & $719 \pm 68$ & & & \\
\hline 0.66 & & & & & & $171 \pm 16$ & \\
\hline 1 & & & & & & $164 \pm 8$ & \\
\hline \multicolumn{8}{|c|}{ 2-Nitrobenzidine } \\
\hline 0 & $23 \pm$ & 9 & $55 \pm 10$ & $23 \pm 5$ & $32 \pm \quad 4$ & $115 \pm 12$ & $130 \pm 22$ \\
\hline 0.01 & & & $81 \pm 14$ & $32 \pm 8$ & $34 \pm \quad 7$ & & \\
\hline 0.05 & & & $218 \pm 17$ & $37 \pm \quad 6$ & $54 \pm \quad 2$ & & \\
\hline 0.1 & $29 \pm$ & 4 & $347 \pm 24$ & $45 \pm \quad 9$ & $93 \pm 23$ & & \\
\hline 0.2 & $46 \pm$ & 5 & $699 \pm 46$ & & & $126 \pm 16$ & $207 \pm 19$ \\
\hline 0.25 & & & & $63 \pm 6$ & $203 \pm 8$ & & \\
\hline 0.4 & $44 \pm$ & 7 & $1617 \pm 120$ & & & $126 \pm 15$ & $299 \pm 42$ \\
\hline 0.5 & $58 \pm 1$ & 16 & $2072 \pm 93$ & $93 \pm 23$ & $403 \pm 80$ & & \\
\hline 0.6 & $67 \pm 1$ & 11 & $2463 \pm 220$ & & & $132 \pm 4$ & $344 \pm 66$ \\
\hline 0.8 & $74 \pm 1$ & 10 & & & & $127 \pm 11$ & $344 \pm 25$ \\
\hline 1 & $86 \pm$ & 8 & & $160 \pm 62$ & $835 \pm 107$ & $149 \pm 12$ & $389 \pm 15$ \\
\hline
\end{tabular}

\footnotetext{
a Combined data from at least two independent experiments, with triplicate plating per dose and at least 5 doses per experiment.
} 
TABLE 2

MUTAGENICITY (REVERTANTS / $\mu$ mole) OF BENZIDINES IN Salmonella typhimurium ${ }^{\text {a }}$

\begin{tabular}{|c|c|c|c|c|c|c|}
\hline \multirow[t]{2}{*}{ Derivative } & \multicolumn{2}{|l|}{ TA98 } & \multicolumn{2}{|c|}{${\text { TA98 } / 1,8-D^{-}}_{6}$} & \multicolumn{2}{|l|}{ TA100 } \\
\hline & $-\mathrm{S} 9$ & $+\mathrm{S} 9$ & $-\mathrm{S} 9$ & + S9 & $-\mathrm{S9}$ & $+\mathrm{S} 9$ \\
\hline Benzidine & 0 & $157 \pm$ & 0 & $4 \pm$ & 0 & $70 \pm \quad 10$ \\
\hline 3,3'-Diamino & $16 \pm$ & $2681 \pm$ & 0 & $856 \pm$ & $84 \pm$ & $768 \pm$ \\
\hline 3,3'-Dimethoxy & 0 & $2905 \pm$ & $21 \pm 3$ & $54 \pm$ & 0 & $69 \pm$ \\
\hline 3,3'-Dimethyl & $11 \pm 4$ & $927 \pm$ & 0 & $215 \pm$ & 0 & $104 \pm \quad 4$ \\
\hline 3,3'-Difluoro & $280 \pm 38$ & $6709 \pm$ & $374 \pm 65$ & $1095 \pm \quad 43$ & 0 & $232 \pm \quad 18$ \\
\hline 3,3'-Dichloro & $7657 \pm 852$ & $25592 \pm$ & $2809 \pm 297$ & $10945 \pm 706$ & 0 & $1351 \pm 67$ \\
\hline 3,3'-Dibromo & $4024 \pm 434$ & $33257 \pm 743$ & $1061 \pm 104$ & $13089 \pm 511$ & 0 & $1102 \pm 60$ \\
\hline 3,3'-Dicarbomethoxy & $192 \pm 41$ & $24954 \pm 1061$ & 0 & $5333 \pm 382$ & 0 & $1046 \pm 152$ \\
\hline 3,3'-Dinitro & $582 \pm 49$ & $355967 \pm 5273$ & 0 & $309353 \pm 8585$ & $3753 \pm 789$ & $111640 \pm 6618$ \\
\hline 3-Nitro & $3969 \pm 171$ & $927423 \pm 16154$ & $1973 \pm 578$ & $140580 \pm 6683$ & $320 \pm 52$ & $30079 \pm 1558$ \\
\hline 2-Nitro & $63 \pm 5$ & $4064 \pm \quad 81$ & $132 \pm 13$ & $807 \pm \quad 27$ & $25 \pm 6$ & $365 \pm 38$ \\
\hline
\end{tabular}

a Values are the calculated slopes (rev/ $\mu$ mole) of the linear portion of the dose-response curve derived from the data in Table 1 .

1987; Sinsheimer et al., 1992). The high mutagenicity of 3-nitrobenzidine is assumed to be caused by activation of the amino group adjacent to the nitro substitution with the other amino group undergoing much weaker long interactions from the nitro group. This assumption is also the basis of our utilization of aniline $\mathrm{p} K \mathrm{Ka}$ values for the relative basicity of the corresponding $3,3^{\prime}$-disubstituted benzidines. The large difference be- tween a 3- and 3'-substituent in their effects on the 4-amino group is illustrated by the $\mathrm{p} \mathrm{Ka}$ values of 4-aminobiphenyl (4.05), 4-amino-3-chlorobiphenyl (2.76) and 4-amino-3'-chlorobiphenyl (3.74) (Byron et al., 1966). Using this approach, the basicity of the 4-amino group in 3-nitrobenzidine is comparable to that of the 4-amino group in 3,3'-dinitrobenzidine.

Mutagenicity in TA98 with S9 activation corre-

TABLE 3

PHYSICOCHEMICAL PARAMETERS FOR THE BENZIDINE SERIES

\begin{tabular}{lrlrrr}
\hline Derivative & $\mathrm{pKa}^{\mathrm{a}}$ & $\mathrm{pK \textrm {a } ^ { \mathrm { b } }}$ & \multicolumn{1}{c}{$E_{\text {HOMO }}{ }^{\mathrm{c}}$} & \multicolumn{1}{c}{$E_{\text {LUMO }}{ }^{\mathrm{c}}$} & $\pi_{\text {HPLC }}{ }^{\mathbf{d}}$ \\
\hline Benzidine & 4.60 & $4.80 \pm 0.08$ & -7.607 & 0.349 & 0.00 \\
3,3'-Diamino & 4.74 & & -7.105 & 0.499 & -0.16 \\
3,3'-Dimethoxy & 4.52 & $4.70 \pm 0.07$ & -7.483 & 0.275 & 0.20 \\
3,3'-Dimethyl & 4.44 & $5.16 \pm 0.05$ & -7.555 & 0.381 & 0.27 \\
3,3'-Difluoro & 3.20 & & -7.884 & -0.055 & 0.21 \\
3,3'-Dichloro & 2.64 & $3.43 \pm 0.03$ & -7.815 & 0.048 & 0.65 \\
3,3'-Dibromo & 2.53 & $3.19 \pm 0.06$ & -7.864 & -0.095 & 0.78 \\
3,3'-Dicarbomethoxy & 2.23 & & -7.868 & -0.173 & 0.75 \\
3,3'-Dinitro & -0.26 & & -8.518 & -0.992 & 0.72 \\
3-Nitro & -0.26 & & -8.004 & -0.737 & -0.78 \\
2-Nitro & & & -7.993 & -0.06
\end{tabular}

a Values from corresponding $o$-substituted anilines (Biggs and Robinson, 1961; Perrin, 1965).

b Values determined experimentally by the spectrophotometric method of Albert and Serjeant (1984).

c Frontier orbital energies calculated as described in Materials and methods.

d Partition coefficients determined by the method of Carlson et al. (1975). 
lated with the basicity of the amines, as measured by the $\mathrm{p} K \mathrm{a}$ values for their corresponding anilines.

$\log ($ TA98 $)=-0.57 \mathrm{p} K \mathrm{a}+5.70$

$n=10, r^{2}=0.89$

Similar correlations were obtained for strains TA98/1,8-DNP 6 and TA100.

$\log \left(\mathrm{TA} 98 / 1,8-\mathrm{DNP}_{6}\right)=-0.72 \mathrm{p} K \mathrm{a}+5.35$

$n=10, r^{2}=0.80$

$\log (\mathrm{TA1} 100)=-0.53 \mathrm{p} K \mathrm{a}+4.48$

$n=10, r^{2}=0.86$

These correlations show a general trend of increasing mutagenicity for the benzidine derivatives with a decrease in their $\mathrm{p} K \mathrm{a}$ values. Since basicity of a substituted aromatic amine must be influenced by electron-donating/withdrawing ability of its substituent, a relationship between mutagenicity and this electronic effect should also exist. The effect of ortho-substituents has been shown to consist of ordinary electronic, proximity electronic and steric effects (Fujita and Nishioka, 1976; Segura, 1985). When the steric factor is negligible, the overall effect of ortho-substituent then becomes electronic in nature. This is essentially true for $o$-substituted anilines, where steric effects accounted for less than $10 \%$ of $\mathrm{pKa}$ change (Fujita and Nishioka, 1976). In this case, relative basicity of the anilines reflects relative electronic effects of $o$-substituents. Also, since the correlations in their study showed a lack of significant hydrogen-bonding disturbance on $\mathrm{p} K \mathrm{a}$, the basicity data of these anilines should be a good approximation of the relative electronwithdrawing ability of the $o$-substituents. Thus our correlations show an overall trend of increasing mutagenicity with an increase in the electron-withdrawing ability of the substituents ortho to the amino group. The most notable

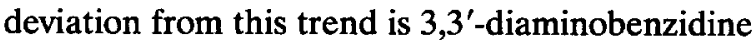
whose regioselectivity for enzymatic activation is unknown and only assumed to be at the 4-amino position.
A structure-mutagenicity study on anilines was reported recently by Kalopissis (1991), which included basicity as only one of the parameters. Our model is different in that we correlate mutagenicity using solely the relative basicities of the benzidines. While both models depend upon basicities, the results are different. For example, we can explain the very high mutagenicity of $3,3^{\prime}$-dinitrobenzidine (very low $\mathrm{p} \mathrm{Ka}$ ) and the relatively low potency of 3,3'-diaminobenzidine (high $\mathrm{p} \mathrm{Ka}$ ) while the Kalopissis model assigns low mutagenicity to 2-nitroaniline and very high potency to 2-aminoaniline.

An examination of the effect of hydrophobicity on mutagenicity shows that there is only a poor correlation between the $\log$ of mutagenicity and $\pi_{\text {HPLC }}$ values $\left(r^{2}=0.40,0.42\right.$ and 0.26 for TA98, TA98 $/ 1,8-\mathrm{DNP}_{6}$ and TA100 respectively). Also, a combination of $\mathrm{pKa}$ and $\pi_{\text {HPLC }}$ data does not or only marginally improves the correlation with mutagenicity over that with $\mathrm{p} K \mathrm{a}$ alone.

Correlation studies of mutagenicity with $E_{\text {LUMo }}$ give similar results to that with $\mathrm{pKa}$.

$\log ($ TA98 $)=-2.10 E_{\text {LUMO }}+3.97$

$n=10, r^{2}=0.80$

$\log \left(\mathrm{TA} 98 / 1,8-\mathrm{DNP}_{6}\right)=-2.60 E_{\text {LUMO }}+3.19$

$n=10, r^{2}=0.70$

$\log ($ TA100 $)=-1.99 E_{\text {LUMO }}+2.87$

$n=10, r^{2}=0.81$

On the other hand, substantially less correlation is obtained for $E_{\text {HOMO }}\left(r^{2}=0.52,0.49\right.$ and 0.53 for TA98, TA98/1,8-DNP 6 and TA100 respectively). A combination of $E_{\text {LUMO }}$ and $\pi_{\text {HPLC }}$ data produces little or marginal improvement over $E_{\text {Lumo }}$ alone.

In the established genotoxicity mechanisms for aromatic amines (Beland and Kadlubar, 1985; $1990)$, the intermediate $N$-hydroxylamine and ultimate DNA binding species, the nitrenium ion (Helmick and Novak, 1991), could serve as the basis of our correlations. There have been a 
number of proposals in this regard based on solubility and stability concepts. Debnath et al. (1992) stressed the importance of hydrophobicity in their Salmonella mutagenicity correlations for a large number of compounds of diverse structures. Our results, however, for a more closely related series of amines showed that mutagenicity was highly dependent on the electronic effects of benzidine substituents and largely independent of the relative partition coefficients of these benzidines.

Ford and Herman (1992) found a limited correlation between Salmonella mutagenicity and the stability of the nitrenium ion for some polycyclic aromatic amines. The more stable the nitrenium ions, the more mutagenic were the corresponding aromatic amines. Since electron-withdrawing groups which increased the mutagenicity of our benzidine derivatives would destabilize the nitrenium ions, this rationale cannot explain our correlations.
The stability of the $N$-hydroxylamines, however, may be used to explain our results in a way similar to that of Vance et al. (1987) for the mutagenicity of 2-nitrofluorene derivatives. $\mathrm{N}$ Hydroxylamines are generally unstable species with strong lone pair-lone pair electron repulsion between the bonding nitrogen and oxygen atoms. Electron-withdrawing groups can reduce such repulsion and help stabilize hydroxylamines, thus allowing longer diffusion paths and an increased probability of interaction with DNA. Destabilization by electron-withdrawing groups of the nitrenium ions may also increase mutagenicity since these ions on one hand should have shorter life times, but on the other, should also be more reactive toward DNA.

\section{In vivo genotoxicity}

Benzidine and its derivatives were also compared for their in vivo genotoxicity where the end point was induced chromosomal aberrations in

TABLE 4

IN VIVO CHROMOSOMAL ABERRATIONS INDUCED BY BENZIDINES AT $100 \mathrm{mg} / \mathrm{kg}$

\begin{tabular}{|c|c|c|c|c|c|}
\hline \multirow[t]{2}{*}{ Derivative } & \multirow[t]{2}{*}{ Gaps $^{\mathbf{a}}$} & \multicolumn{2}{|c|}{ Aberrations/cell } & \multirow{2}{*}{$\begin{array}{l}\text { Aberrant cells }(\%)^{b} \\
(\text { mean } \pm \text { S.D. })^{c}\end{array}$} & \multirow{2}{*}{$\begin{array}{l}\text { Mitotic indices (\%) } \\
(\text { mean } \pm \text { S.D. })^{c}\end{array}$} \\
\hline & & $\begin{array}{l}\text { Chromatid } \\
\text { type }\end{array}$ & $\begin{array}{l}\text { Chromosome } \\
\text { type }\end{array}$ & & \\
\hline $\begin{array}{l}\text { Solvent control } \\
\text { DMSO }(2 \mathrm{ml} / \mathrm{kg})\end{array}$ & 3.00 & 0.010 & 0.000 & $1.00 \pm 0.82$ & $3.51 \pm 0.30$ \\
\hline $\begin{array}{l}\text { Positive control } \\
\text { DMBA }\end{array}$ & 11.25 & 0.115 & 0.023 & $13.25 \pm 0.96 * *$ & $2.13 \pm 0.12 * *$ \\
\hline $\begin{array}{l}\text { Benzidine } \\
\text { 3,3'-Diamino } \\
\text { 3,3'-Dimethoxy }\end{array}$ & $\begin{array}{l}6.25 \\
4.25 \\
4.25\end{array}$ & $\begin{array}{l}0.043 \\
0.043 \\
0.043\end{array}$ & $\begin{array}{l}0.080 \\
0.000 \\
0.010\end{array}$ & $\begin{array}{l}5.00 \pm 0.82 * * \\
4.25 \pm 0.50 * * \\
5.25 \pm 0.96 * *\end{array}$ & $\begin{array}{l}2.63 \pm 0.33 * * \\
2.43 \pm 0.28 * * \\
2.05 \pm 0.15 * *\end{array}$ \\
\hline $\begin{array}{l}\text { 3,3'-Dimethyl } \\
\text { 3,3'-Difluoro } \\
\text { 3,3'-Dichloro }\end{array}$ & $\begin{array}{l}3.00 \\
2.75 \\
4.25\end{array}$ & $\begin{array}{l}0.025 \\
0.023 \\
0.028\end{array}$ & $\begin{array}{l}0.008 \\
0.003 \\
0.003\end{array}$ & $\begin{array}{l}3.25 \pm 0.50 * * \\
2.50 \pm 0.58 * \\
3.00 \pm 0.82 * *\end{array}$ & $\begin{array}{l}2.38 \pm 0.29 * * \\
2.98 \pm 0.30 * \\
2.37 \pm 0.25 * *\end{array}$ \\
\hline $\begin{array}{l}\text { 3,3'-Dibromo } \\
\text { 3,3'-Dicarbomethoxy } \\
\text { 3,3'-Dinitro }\end{array}$ & $\begin{array}{l}2.50 \\
3.50 \\
6.75\end{array}$ & $\begin{array}{l}0.028 \\
0.025 \\
0.053\end{array}$ & $\begin{array}{l}0.000 \\
0.003 \\
0.005\end{array}$ & $\begin{array}{l}2.75 \pm 0.50 * * \\
2.75 \pm 0.96 * \\
5.75 \pm 0.96 * *\end{array}$ & $\begin{array}{l}3.01 \pm 0.35 * \\
2.77 \pm 0.45 * \\
2.14 \pm 0.19 * *\end{array}$ \\
\hline $\begin{array}{l}\text { 3-Nitro } \\
\text { 2-Nitro }\end{array}$ & $\begin{array}{l}6.50 \\
5.75\end{array}$ & $\begin{array}{l}0.068 \\
0.065\end{array}$ & $\begin{array}{l}0.010 \\
0.000\end{array}$ & $\begin{array}{l}7.75 \pm 0.96 * * \\
6.50 \pm 1.00 * *\end{array}$ & $\begin{array}{l}2.26 \pm 0.24 * * \\
2.50 \pm 0.30 * *\end{array}$ \\
\hline
\end{tabular}

a Total chromatid and chromosome gaps at each concentration per 100 cells were recorded but not included as aberrations.

b Cells with at least 1 aberration for 4 animals.

c Results were compared to those of the control, where statistically significant differences are indicated by ${ }^{*} P<0.05$ and ** $P<0.01$ by Student's $t$ test. 
the bone-marrow cells of mice after i.p. injection of the amines. A comparison of the in vivo results is summarized in Table 4. At a dose of 100 $\mathrm{mg} / \mathrm{kg}$, a statistically significant increase in genotoxicity was established for all the compounds in comparison to the DMSO negative control. However, none of the compounds approached the genotoxicity of the positive control, DMBA. The relative genotoxicity in vivo did not correlate to our in vitro mutagenicity data. While the 3,3-dinitro- and 3-nitro-benzidine derivatives were again among the most genotoxic, it was only for the 3-nitro derivative that a statistically significant increase in genotoxicity over that for benzidine could be established. In contrast to the in vitro results, 2-nitrobenzidine and 3,3'-dimethoxybenzidine were among the more genotoxic compounds and the halogenated and carbomethoxy derivatives were significantly less genotoxic than benzidine.

It is of interest that the relative genotoxicity of benzidine, which was the least mutagenic compound in vitro, exhibits genotoxicity generally greater or at least equal to the other compounds in vivo. This might be explained by the established role of $N$-acetylation of one amino group prior to activation and DNA binding of benzidine in vivo (Beland and Kadlubar, 1985, 1990). This activation pathway was limited under our in vitro conditions (Kennelly et al., 1984). Also, the multiple metabolic routes for the activation of a hydroxylamine by esterification prior to nitrenium ion formation available in vivo (Beland and Kadlubar, 1985) but absent under our in vitro conditions (Weeks et al., 1978; Wirth and Thorgeirsson, 1980) would contribute to the differences in genotoxicity in vivo vs. in vitro. An additional potential metabolic activation pathway that could play a role in the genotoxicity of benzidine compounds in vivo but that would be absent under our in vitro conditions would be oxidation by peroxidase enzymes (Josephy, 1986; Kadlubar et al., 1986). Thus, while it is reasonable to assume that the substituents have similar effects on the stability and activity of $N$-hydroxylamines as well as nitrenium ions in vivo as in vitro, the differences between the two systems such as longer diffusion paths and different metabolic pathways of amine activation and detoxication for in vivo vs. in vitro could account for the differences between these two series of genotoxicity results.

\section{Acknowledgement}

This investigation was supported by grant RO1 ES05047 from the National Institute of Environmental Health Sciences, DHHS.

\section{References}

Albert, A., and E.P. Serjeant (1984) The Determination of Ionization Constants, A Laboratory Manual, 3rd edn., Chapman and Hall, New York.

Barker, C.C., and F.D. Casson (1953) The preparation and deamination of $4: 4^{\prime}$-diamino-3:3'-dinitrodiphenyl, $\mathrm{J}$. Chem. Soc., 4184-4185.

Beland, F.A., and F.F. Kadlubar (1985) Formation and persistence of arylamine DNA adducts in vivo, Environ. Health Perspect., 62, 19-30.

Beland, F.A., and F.F. Kadlubar (1990) Metabolic activation and DNA adducts of aromatic amines and nitroaromatic hydrocarbons, in: C.S. Cooper and P.L. Grover (Eds.), Chemical Carcinogenesis and Mutagenesis I, Springer, Berlin, pp. 267-325.

Bernstein, L., J. Kaldor, J. McCann and M.C. Pike (1982) An empirical approach to the statistical analysis of mutagenesis data from the Salmonella test, Mutation Res., 97, 267-281.

Biggs, A.I., and R.A. Robinson (1961) The ionisation constants of some substituted anilines and phenols: a test of the Hammett Relation, J. Chem. Soc., 388-393.

Buckingham, J. (1982) Dictionary of Organic Compounds, 5th edn., Chapman and Hall, New York.

Byron, D.J., G.W. Gray and R.C. Wilson (1966) The effects of $3^{\prime}$ and $4^{\prime}$ substituents on the ionization constants of biphenyl-4-carboxylic acid and 4-aminobiphenyl, J. Chem. Soc. (C), 831-836.

Carlson, R.M., R.E. Carlson and H.L. Kopperman (1975) Determination of partition coefficients by liquid chromatography, J. Chromatogr., 107, 219-223.

Debnath, A.K., G. Debnath, A.J. Shusterman and C. Hansch (1992) A QSAR investigation of the role of hydrophobicity in regulating mutagenicity in the Ames test, 1. Mutagenicity of aromatic and heteroaromatic amines in Salmonella typhimurium TA98 and TA100, Environ. Mol. Mutagen., $19,37-52$.

Ford, G.P., and G.R. Griffin (1992) Relative stabilities of nitrenium ions derived from heterocyclic amine food carcinogens: Relationship to mutagenicity, Chem.-Biol. Interact., 81, 19-33.

Ford, G.P., and P.S. Herman (1992) Relative stabilities of nitrenium ions derived from polycyclic aromatic amines. Relationship to mutagenicity, Chem.-Biol. Interact., 81 , $1-18$. 
Fujita, T., and T. Nishioka (1976) The analysis of the ortho effect, in R.W. Taft (Ed.), Prog. in Phys. Org. Chem., Vol. 12, Wiley, New York, pp. 49-89.

Giri, A.K., E.A. Messerly and J.E. Sinsheimer (1989) Sisterchromatid exchange and chromosome aberrations for 4 aliphatic epoxides in mice, Mutation Res., 224, 253-261.

Helmick, J.S., and M. Novak (1991) Nucleophilic substitution at nitrogen and carboxyl carbon of $N$-aryl- $O$-pivaloylhydroxylamines in aqueous solution: Competition with $S_{N} 1$ solvolysis of model carcinogens, J. Org. Chem., 56, 29252927.

Iwakura, Y., K. Uno and N. Chau (1973) Synthesis of poly(amide-quinazolinedione)s, Polymer J., 5, 301-308.

Josephy, P.D. (1986) Benzidine: mechanisms of oxidative activation and mutagenesis, Fed. Proc., 45, 2465-2470.

Kadlubar, F.F., Y. Yamazoe, N.P. Lang, D.Z.J. Chu and F.A. Beland (1986) Carcinogen-DNA adduct formation as a predictor of metabolic activation pathways and reactive intermediates in benzidine carcinogenesis, Adv. Exp. Med. Biol., 197, 537-549.

Kalopissis, G. (1991) Structure-activity relationships of aromatic amines in the Ames Salmonella typhimurium assay, Mutation Res., 246, 45-66.

Kennelly, J.C., C.A. Stanton and C.N. Martin (1984) The effect of acetyl-CoA supplementation on the mutagenicity of benzidines in the Ames assay, Mutation Res., 137, 39-45.

Leslie, M.S., and E.E. Turner (1933) The optical activity of a diphenyl derivative, the dissymmetry of which is caused by the space effect of only one group, J. Chem. Soc., 15881592.

Maron, D.M., and B.N. Ames (1983) Revised methods for the Salmonella mutagenicity test, Mutation Res., 113, 173-215.

Messerley, E.A., J.E. Fekete, D.R. Wade and J.E. Sinsheimer (1987) Structure-mutagenicity relationships of benzidine analogues, Environ. Mol. Mutagen., 10, 263-274.

Mysyk, D.D. (1976) Acyl derivatives of arylenediamines, VI. Synthesis and nitration of aryl-substituted 4,4'-diaminobiphenyls, J. Org. Chem. (U.S.S.R.), 12, 2144-2147.

Orr, J.C., D.W. Bryant, D.R. McCalla and M.A. Quillam (1985) Dinitropyrene-resistant Salmonella typhimurium are deficient in an acetyl-CoA acetyltransferase, Chem.-Biol. Interact., 54, 281-288.

Perrin, D.D. (1965) Dissociation Constants of Organic Bases in Aqueous Solution, Butterworth, London.

Preston, R.J., B.J. Dean, S. Galloway, H. Holden, A.F. McFee and M. Shelby (1987) Mammalian in vivo cytogenetic assays, Analysis of chromosome aberrations in bone-marrow cells, Mutation Res., 189, 157-165.

Sabbioni, G. (1992) Hemoglobin binding of monocyclic aromatic amines: Molecular dosimetry and quantitative structure activity relationships for the $\mathrm{N}$-oxidation, Chem.-Biol. Interact., 81, 91-117.
Saito, K., A. Shinohara, T. Kamataki and R. Kato (1985) Metabolic activation of mutagenic $N$-hydroxyarylamines by $O$-acetyltransferase in Salmonella typhimurium TA98, Arch. Biochem. Biophys., 239, 286-295.

Savard S., and P.D. Josephy (1986) Synthesis and mutagenicity of 3,3'-dihalogenated benzidines, Carcinogenesis, 7 , 1239-1241.

Segura, P. (1985) Simultaneous use of empirical and semiempirical substituent parameters as a new method of analysis of the ortho effect, Application in reactions via aryl anion intermediates, J. Org. Chem., 50, 1045-1053.

Sinsheimer, J.E., B.H. Hooberman, S.K. Das, M.D. Brezzell and Z. You (1992) The in vivo and in vitro genotoxicity of aromatic amines in relationship to the genotoxicity of benzidine, Mutation Res., 268, 255-264.

Snyder, H.R., C. Weaver and C.D. Marshall (1949) The preparation of $3,3^{\prime}$-dibromobiphenyl and its conversion to organometallic compounds, J. Am. Chem. Soc., 71, 289291.

Steward, J.J.P. (1989a) Optimization of parameters for semiempirical methods, I. Method, J. Comp. Chem., 10, 209-220.

Steward, J.J.P. (1989b) Optimization of parameters for semiempirical methods, II. Applications, J. Comp. Chem., 10, 221-264.

Vance, W.A., Y.Y. Wang and H.S. Okamoto (1987) Disubstituted amino-, nitroso-, and nitrofluorenes: A physicochemical basis for structure-activity relationships in Salmonella typhimurium, Environ. Mutagen., 9, 123-141.

Weast, R.C., and J.G. Grasselli (1989) Handbook of Data on Organic Compounds, 2nd edn., CRC Press, Boca Raton.

Weeks, C.E., W.T. Allaben, S.C. Louie, E.J. Lazear and C.M. King (1978) Role of arylhydroxamic acid acyltransferase in the mutagenicity of $N$-hydroxy- $N$-2-fluorenylacetamide in Salmonella typhimurium, Cancer Res., 38, 613-618.

Weisburger, E.K., and S.S. Thorgeirsson (1981) Carcinogenic and mutagenic $N$-substituted aryl compounds, NIH Publ. No. 81-2379, National Cancer Institute, Bethesda.

Wirth, P.J., and S.S. Thorgeirsson (1980) Mechanism of $N$-hydroxy-2-acetylaminofluorene mutagenicity in the Salmonella test system, Role of $N-O$ acyltransferase and sulfotransferase from rat liver, Mol. Pharmacol., 19, 337-344.

You, Z., M.D. Brezzell, S.K. Das, B.H. Hooberman and J.E. Sinsheimer (1993) Substituent effects on the in vitro and in vivo genotoxicity of 4-aminobiphenyl and 4-aminostilbene derivatives, in preparation.

Zheltov, A.Y., V.Y. Rodionov and B.I. Stepanov (1970) Investigations on aromatic disulfides, III. Basicity and structure of substituted 4- and 5-aminobiphenylene-2,2'-disulfide, J. Org. Chem. (U.S.S.R.), 6, 2573-2578. 